\title{
molecules
}

ISSN 1420-3049

http://www.mdpi.org

Full Paper

\section{Functionalisation of Artemisinin and Its Ring-contracted Derivatives}

\section{Tine Van Neck, Sarah Van Mierloo and Wim Dehaen*}

Department of Chemistry, University of Leuven, Celestijnenlaan 200F B-3001 Leuven, Belgium

* Author to whom correspondence should be addressed; E-mail: wim.dehaen@chem.kuleuven.be; Tel. (+32) 163274 39; Fax (+32) 16327990

Received: 1 March 2007; in revised form: 8 March 2007 / Accepted: 8 March 2007 /

Published: 9 March 2007

\begin{abstract}
Isoxazoline analogues of artemisinin were obtained in low yield and low diastereoselectivity from the 1,3-dipolar cycloaddition of nitrile oxides. Alternatively, starting from the aldehyde 7 , a number of transformations - Wittig reaction and reduction, Henry reaction and cyanohydrin formation - were achieved in significantly higher yields. In the cases where a new stereocenter was introduced this occured diastereoselectively.
\end{abstract}

Keywords: Artemisinin, ring contraction, aldehyde, 1,3-dipolar cycloaddition, nitrile oxide.

\section{Introduction}

Artemisinin (1, qinghaosu, arteannuin) is a potent antimalarial agent with an interesting trioxane sesquiterpene structure, and has been isolated from the plant Artemisia annua. This plant has been used in Traditional Chinese Medicine for nearly 2000 years as a cure for malaria. Great efforts have been focused on chemical modifications of the artemisinin structure in order to improve the biological activity. Semisynthetic analogues of $\mathbf{1}$, such as the lactol dihydroartemisinin (2) and its ether derivatives $\beta$-artemether (3), $\beta$-arteether (4) and sodium artesunate (5) have received significant attention in this context [1]. 


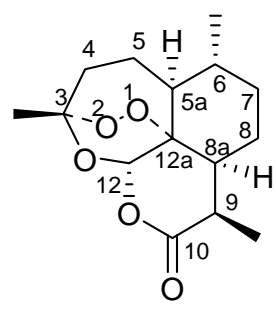

Artemisinin 1

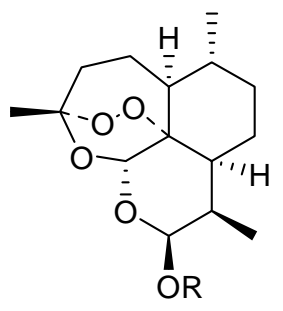

$2 \mathrm{R}=\mathrm{H}$
$3 \mathrm{R}=\mathrm{Me}$
$4 \mathrm{R}=\mathrm{Et}$
$5 \mathrm{R}=\mathrm{COCH}_{2} \mathrm{CH}_{2} \mathrm{COONa}$

One problem is the metabolic instability of the ethers 3-5 and their analogs, that undergo fast transformation into the neurotoxic 2 . We propose a synthetic exploration of the known carbonconnected artemisinin derivatives 6 [2] and 7 [3] to prepare a variety of derivatives by 1,3-dipolar cycloaddition or other reactions.
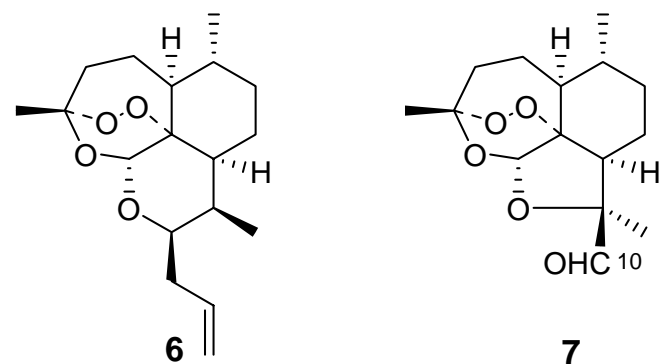

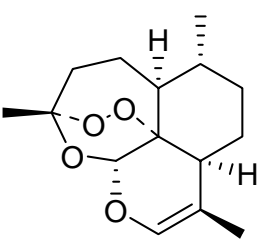

8

\section{Results and Discussion}

The $\beta$-allyl derivative $\mathbf{6}$ was prepared in good yield starting from the benzoate of $\mathbf{2}$ using the literature procedure [2]. The dehydrodeoxy analog 8 was formed at the same time as a side product (10 $\%)$. The acetate analog of $\mathbf{2}$, in comparison, gave much less of the desired $\mathbf{6}$, vinyl ether $\mathbf{8}$ being the major product (50\%). Compound 6 was then used as a dipolarophile in combination with in situ generated [4] benzonitrile oxide. A cycloadduct 9 was isolated in $29 \%$ yield after careful chromatography, and from NMR-analysis it was found to be a 60:40 mixture of diastereoisomers (stereochemistry not assigned) (Scheme 1). The vinyl ether $\mathbf{8}$, when combined with benzonitrile oxide under the same conditions, did not afford an isoxazoline adduct, but rather the starting material was recovered.

\section{Scheme 1.}

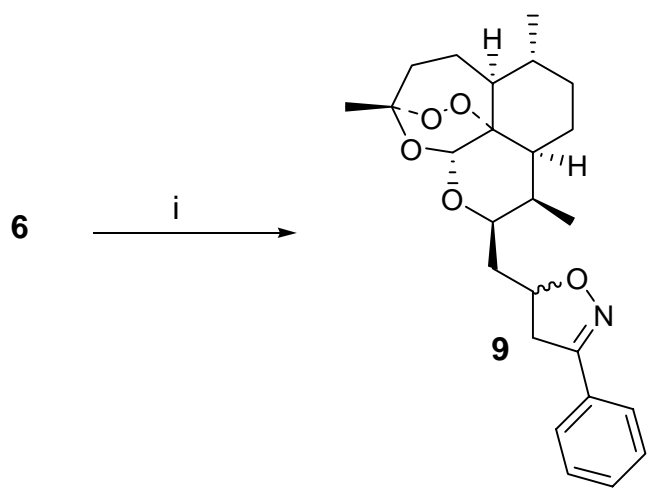

i) $\mathrm{PhCH}=\mathrm{NOH}, \mathrm{NaOCl}, \mathrm{Et}_{3} \mathrm{~N}, \mathrm{CH}_{2} \mathrm{Cl}_{2}, \mathrm{RT}$ 
In view of the low yield of isoxazoline 9, the lack of diastereoselectivity and the fact that $\mathbf{6}$ has been previously and extensively used by others [5] to prepare carbon-connected artemisinin derivatives, we decided to shift our attention to the ring-contracted artemisinin derivative 7. This compound is readily available starting from the dehydro analog $\mathbf{8}$ by treatment with iodine and rearrangement in polar medium [3]. In the first step we converted this aldehyde 7 into the oxime derivative 10. This oxime $\mathbf{1 0}$ was then used to generate the nitrile oxide intermediate in the presence of excess styrene, affording 1,3-dipolar cycloadduct $\mathbf{1 1}$ in $40 \%$ yield as a 1:1 mixture of diastereoisomers. The diastereomer issue could be avoided when phenylacetylene was used as the dipolarophile. In this case, $21 \%$ of the isoxazole 12 was obtained. The reaction is sensitive to steric hindrance. For instance, use of cyclohexene as the dipolarophile did not lead to the isolation of a cycloadduct and rather a mixture of unidentified compounds was formed (Scheme 2)

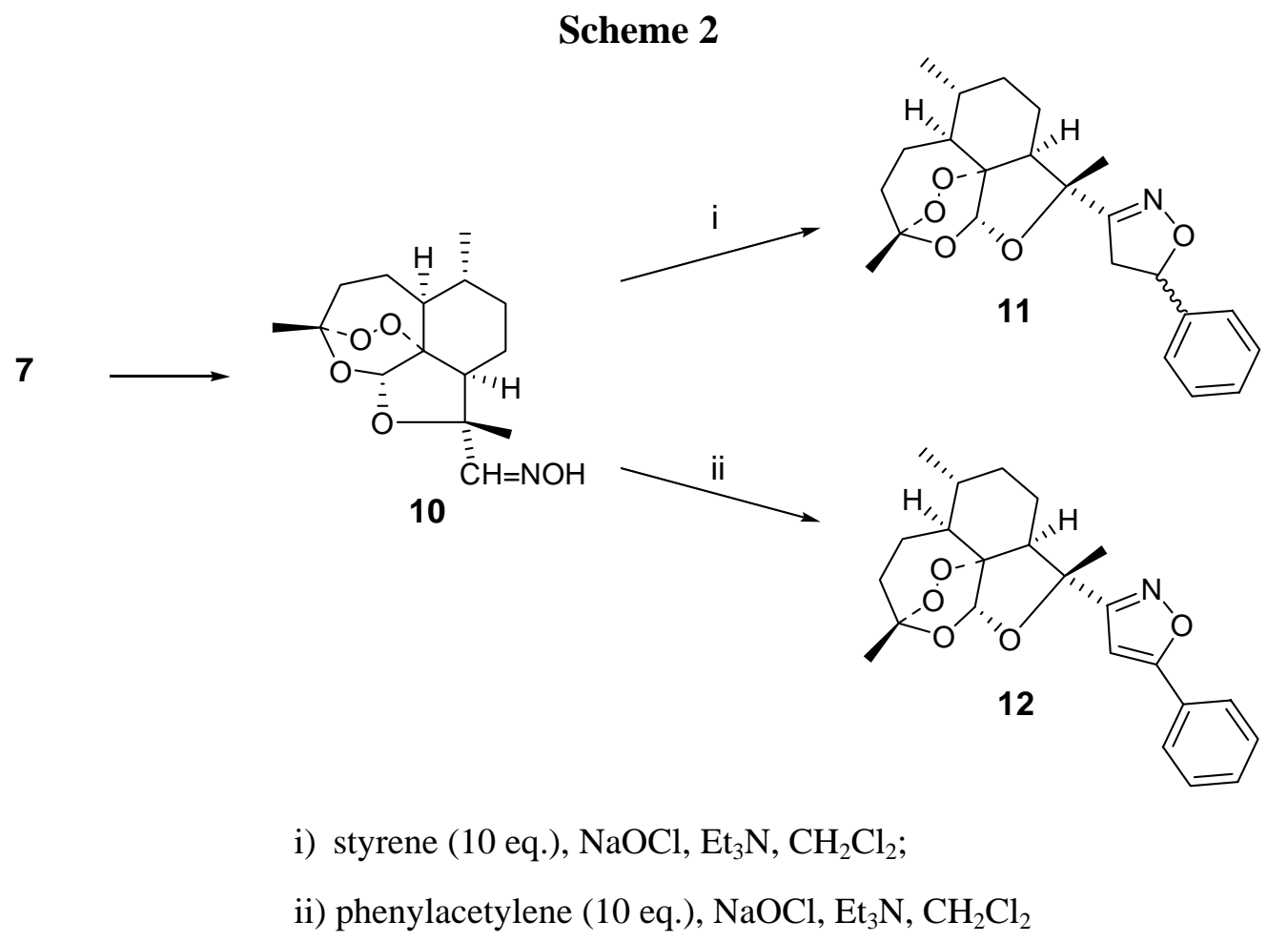

We decided to use the aldehyde 7 to introduce synthetic diversity, extending the functional group away from the artemisinin moiety, as a possible way to increase the reactivity. The Wittig reaction of 7 with benzylidene triphenylphosphorane gave a good yield of the corresponding ( $E$-)-styrene derivative 13. Analogously, Wittig reaction of 7 gave the $(E)$-acrylate ester 14. Remarkably, 14 could be catalytically reduced to the propanol derivative 15 in $74 \%$ yield without cleavage of the peroxide bridge. A reaction time of $12 \mathrm{~h}$ was optimal and longer reaction times resulted in partial decomposition. (Scheme 3) 
Scheme 3.

7

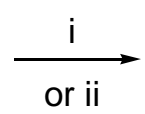

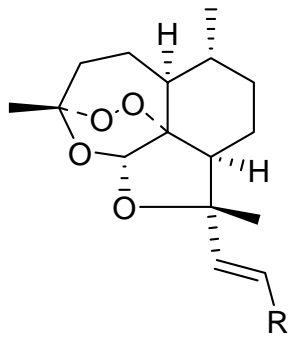

$13 \mathrm{R}=\mathrm{Ph}$

$14 \mathrm{R}=$ COOEt

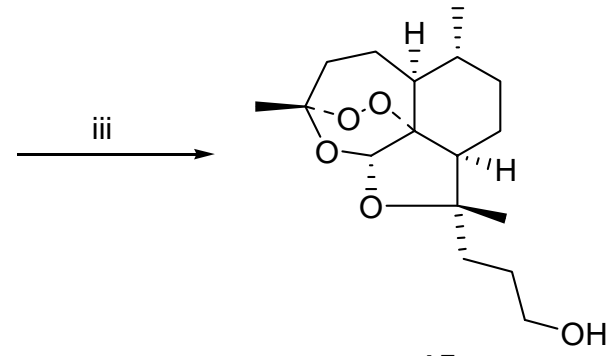

15

i) $\mathrm{PhCH}_{2} \mathrm{PPh}_{3} \mathrm{Br}, \mathrm{BuLi}$, THF, reflux ; ii) EtOOCCH=PPh 3 , RT, THF, overnight; iii) $\mathrm{Pd} / \mathrm{C}(5 \%), \mathrm{H}_{2}$, THF, RT, $12 \mathrm{~h}$

The methanol analogue 16 was prepared from 7 by reduction with excess sodium borohydride. Similar reactions were carried out by Venugopalan on the epimer [6]. Reaction of $\mathbf{1 6}$ with phenyl isocyanate and allyl isothiocyanate resulted in the formation of the urea derivative $\mathbf{1 7}$ or thiourea derivative 18, respectively. Reductive amination of $\mathbf{7}$ with 4-methylaniline afforded a good yield of the secondary aniline 19. Oxidation of 7, on the other hand, gave the carboxylic acid 20. All reactions leading to 16-20 occurred in good yields (53-84 \%) (Scheme 4).

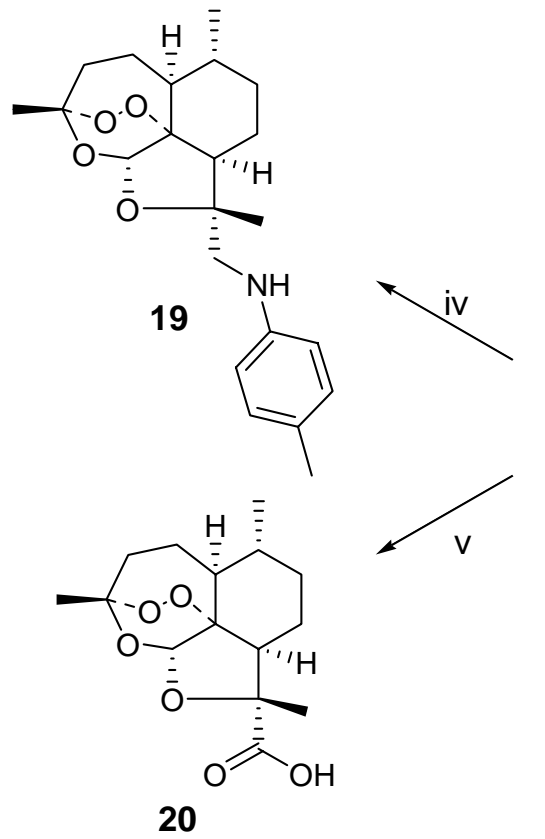

\section{Scheme 4.}

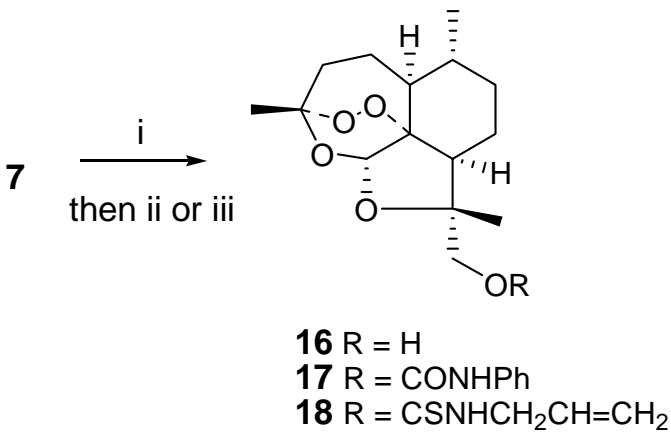

i) $\mathrm{NaBH}_{4}$ (4eq.), methanol, $0^{\circ} \mathrm{C}$, $1 \mathrm{~h}$; ii) phenyl isocyanate, pyridine, $\mathrm{RT}$, $14 \mathrm{~h}$; iii) allyl isothiocyanate, pyridine, $60^{\circ} \mathrm{C}, 14 \mathrm{~h}$; iv) 4-methylaniline (4eq.), $\mathrm{NaBH}_{4}$ (4eq.), methanol, RT, 30 min.; v) aminosulfonic acid (4 eq.), sodium chlorite (4 eq.), water/dioxane, RT, $30 \mathrm{~min}$.

Finally, we studied nucleophilic additions to aldehyde $\mathbf{7}$ as a way to introduce synthetic diversity. Earlier trifluoromethyl and enolate anions had been used [3] to prepare ketone derivatives of $\mathbf{7}$. We 
reacted nitromethane and base with 7 to obtain the nitroaldol 21 in $67 \%$ yield. Analogously, cyanide could be used as the nucleophile in the presence or absence of ammonia, affording aminoacetonitrile $\mathbf{2 2}$ or cyanohydrin $\mathbf{2 3}$ in 78 and 83 \% yields, respectively. Only one of two possible diastereoisomers is obtained for 21-23, but the absolute stereochemistry of the new stereocenters has not yet been determined (Scheme 5).

\section{Scheme 5.}

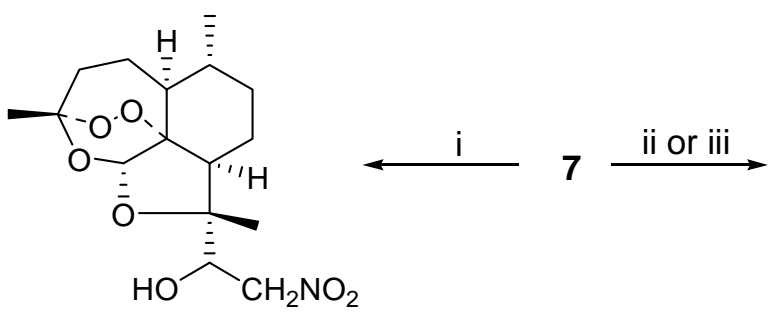

21

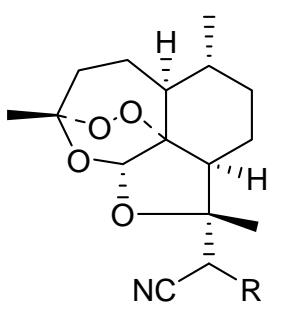

$22 \mathrm{R}=\mathrm{NH}_{2}$ $23 \mathrm{R}=\mathrm{OH}$

i) nitromethane (4 eq.), $\mathrm{NaOMe}$ (excess), ethanol, $0^{\circ} \mathrm{C}, 18 \mathrm{~h}$; ii) $\mathrm{NaCN}$ (5 eq.), $\mathrm{NH}_{4} \mathrm{Cl}$, methanol/water, $45^{\circ} \mathrm{C}$, 2h; iii) $\mathrm{KCN}$ (4 eq.), methanol, acetic acid, $18 \mathrm{~h}$, RT

\section{Conclusions}

Isoxazole analogues of artemisinin can be obtained in low yield and diastereoselectivity, and the cycloaddition reactions used are sensitive to steric hindrance. A number of transformations were carried out in good yields (and diastereoselectivity where appropriate), starting from the ring contracted aldehyde 7 . We hope the novel artemisinin derivatives obtained can be tested in the future for their biological activities.

\section{Experimental}

\section{General}

${ }^{1} \mathrm{H}$ - and ${ }^{13} \mathrm{C}$-NMR spectra were recorded on Varian Mercury $300 \mathrm{MHz}$, Bruker Avance $300 \mathrm{MHz}$, or Bruker AMX3 400 spectrometers using tetramethylsilane as an internal standard. ES MS were measured on Micromass Mattro II instrument. Melting points were determined with a Boetius Block apparatus and are uncorrected. Compounds 6 [2] and 7 [3] were prepared according to the literature procedures.

\section{0-((3-Phenyl-4,5-dihydroisoxazolyl)methyldeoxoartemisinin (9)}

To allyldeoxoartemisinin $6(0.100 \mathrm{~g}, 0.32 \mathrm{mmol})$ in $\mathrm{CH}_{2} \mathrm{Cl}_{2}(5 \mathrm{~mL})$ was added benzaldehyde oxime $(0.2 \mathrm{~mL}, 1.6 \mathrm{mmol})$, a solution of sodium hypochlorite $(0.5 \mathrm{~mL}, 13 \%$ active chlorine, 6.8 mmol) and a few drops of triethylamine. The mixture was stirred overnight at room temperature and afterwards washed with water $(2 \times 10 \mathrm{~mL})$. The organic layer was dried $\left(\mathrm{MgSO}_{4}\right)$ and evaporated to give a residue that was chromatographed over silica gel with $\mathrm{CH}_{2} \mathrm{Cl}_{2}$ as the eluent. The oily product 9 
was obtained as a mixture of diastereoisomers (0.040 g, $29 \%$ ); MS (ES): $428\left(\mathrm{M}^{+}\right)$; Major isomer: ${ }^{1} \mathrm{H}-$ NMR (400 MHz, $\mathrm{C}_{6} \mathrm{D}_{6}, \delta$ ): 0.88 (3H, d, $J=7 \mathrm{~Hz}, \mathrm{CH}_{3}-9$ ), 0.97 (3H, d, $J=3 \mathrm{~Hz}, \mathrm{CH}_{3}-6$ ), 1.26-2.34 (12H, m), 2.69 (1H, m, H-9), 3.13 (1H, dd, $J=9,16 \mathrm{~Hz}$, isoxazolo-H-4), $3.48(1 \mathrm{H}, \mathrm{dd}, J=10,16 \mathrm{~Hz}$, oxazolo-H-4), 4.53 (1H, ddd, $J=2,6,10 \mathrm{~Hz}, \mathrm{H}-10), 4.90$ (1H, m, oxazolo-H-5), 5.33 (1H, s, H-12), 7.38-7.42 (5H, m, Ph); ${ }^{13} \mathrm{C}-\mathrm{NMR}\left(100 \mathrm{MHz}, \mathrm{C}_{6} \mathrm{D}_{6}, \delta\right)$ : $12.7\left(\mathrm{CH}_{3}\right), 20.1\left(\mathrm{CH}_{3}\right), 26.0\left(\mathrm{CH}_{3}\right), 30.3(\mathrm{CH})$, $37.6(\mathrm{CH}), 40.6\left(\mathrm{CH}_{2}\right), 44.1(\mathrm{CH}), 52.2(\mathrm{CH}), 72.0(\mathrm{CH}), 80.3(\mathrm{CH}), 80.9(\mathrm{C}), 89.3(\mathrm{CH}), 103.1(\mathrm{C})$, 126.7 (2xCH), 128.6 (2xCH), $129.9(\mathrm{CH}), 156.9(\mathrm{C})$. Minor isomer: ${ }^{1} \mathrm{H}-\mathrm{NMR}\left(400 \mathrm{MHz}, \mathrm{C}_{6} \mathrm{D}_{6}, \delta\right)$ : 0.88 (3H, d, $J=7 \mathrm{~Hz}, \mathrm{CH}_{3}-9$ ), 0.96 (3H, d, $\left.J=3 \mathrm{~Hz}, \mathrm{CH}_{3}-6\right), 1.26-2.34$ (12H, m), 2.69 (1H, m, H-9), $3.13(1 \mathrm{H}, \mathrm{dd}, J=9,16 \mathrm{~Hz}$, isoxazolo-H-4), 3.42 (1H, dd, $J=10,16 \mathrm{~Hz}$, oxazolo-H-4), 4.35 (1H, ddd, $J=$ 2, 6, $10 \mathrm{~Hz}, \mathrm{H}-10), 5.04$ (1H, m, oxazolo-H-5), 5.37 (1H, s, H-12), 7.38-7.42 (5H, m, Ph); ${ }^{13} \mathrm{C}-$ NMR (100 MHz, C6 $\left.\mathrm{D}_{6}, \delta\right): 12.9\left(\mathrm{CH}_{3}\right), 20.1\left(\mathrm{CH}_{3}\right), 26.0\left(\mathrm{CH}_{3}\right), 30.3(\mathrm{CH}), 37.5(\mathrm{CH}), 39.1\left(\mathrm{CH}_{2}\right)$, $44.2(\mathrm{CH}), 52.3(\mathrm{CH}), 71.5(\mathrm{CH}), 79.9(\mathrm{CH}), 80.9(\mathrm{C}), 89.3(\mathrm{CH}), 103.1(\mathrm{C}), 126.7(2 \times \mathrm{CH}), 128.6$ (2xCH), $129.9(\mathrm{CH}), 156.4(\mathrm{C})$

$(1 S, 4 R, 5 S, 8 R, 9 S, 11 S, 12 S)-1,5,9-T r i m e t h y l-10,13,14,15$-tetraoxatetracyclo[9.1.3.1. $\left.0^{4,12} \cdot 0^{8,12}\right]$ pentadecane-9 $\alpha$-carbaldehyde oxime (10)

To the aldehyde $7(1.00 \mathrm{~g}, 3.5 \mathrm{mmol})$ in methanol $(10 \mathrm{~mL})$ was added hydroxylamine hydrochloride (1.17 g, $16.5 \mathrm{mmol}$ ) and $\mathrm{NaHCO}_{3}(1.39 \mathrm{~g}, 16.5 \mathrm{mmol})$. The mixture was stirred overnight at room temperature and afterwards diluted with water and extracted with dichloromethane ( $3 \mathrm{x} 30 \mathrm{~mL}$ ). The organic layer was dried $\left(\mathrm{MgSO}_{4}\right)$ and evaporated to give a yellow oil (0.84 g, 86 \%); MS (ES): $298\left(\mathrm{M}^{+}\right.$); ${ }^{1} \mathrm{H}-\mathrm{NMR}\left(300 \mathrm{MHz}, \mathrm{CDCl}_{3}, \delta\right): 0.99$ (3H, d, $J=6 \mathrm{~Hz}, \mathrm{CH}_{3}-9$ ), 1.26-2.34 (15H, m), 5.69 (1H, s, C-12), 7.48 (1H, s, OH), 8.05 (1H, s, H-10); ${ }^{13} \mathrm{C}-\mathrm{NMR}\left(75 \mathrm{MHz}, \mathrm{CDCl}_{3}, \delta\right): 14.7\left(\mathrm{CH}_{3}\right), 19.8$ $\left(\mathrm{CH}_{3}\right), 24.6\left(\mathrm{CH}_{2}\right), 25.3\left(\mathrm{CH}_{2}\right), 25.5\left(\mathrm{CH}_{3}\right), 32.5\left(\mathrm{CH}_{2}\right), 36.9(\mathrm{CH}), 46.2(\mathrm{CH}), 48.2(\mathrm{CH}), 84.11(\mathrm{C})$, $86.8(\mathrm{C}), 96.7(\mathrm{CH}), 103.7(\mathrm{C}), 159.6(\mathrm{CH})$.

9- $\alpha$-(5-Phenyl-4,5-dihydrooxazol-3-yl)-(1S,4R,5S,8R,9S,11S,12S)-1,5,9-trimethyl-10,13,14,15-tetraoxatetracyclo[9.1.3.1. $\left.0^{4,12} \cdot 0^{8,12}\right]$ pentadecane (11)

To the oxime 10 (43 mg, $0.15 \mathrm{mmol})$ in $\mathrm{CH}_{2} \mathrm{Cl}_{2}(5 \mathrm{~mL})$ was added styrene $(0.17 \mathrm{~mL}, 1.45 \mathrm{mmol})$, a solution of sodium hypochlorite $(0.5 \mathrm{~mL}, 13 \%$ active chlorine, $6.8 \mathrm{mmol})$ and a few drops of triethylamine. The mixture was stirred overnight at room temperature and afterwards washed with water (2 times $10 \mathrm{~mL}$ ). The organic layer was dried $\left(\mathrm{MgSO}_{4}\right)$ and evaporated to give a residue that was chromatographed over silica gel with dichloromethane as the eluent. The oily product $\mathbf{1 1}$ was obtained as a mixture of diastereoisomers (0.023 g, $40 \%)$; MS (ES): $400\left(\mathrm{M}^{+}\right)$; Major isomer: ${ }^{1} \mathrm{H}-\mathrm{NMR}$ (400 $\left.\mathrm{MHz}, \mathrm{CDCl}_{3}, \delta\right): 1.01\left(6 \mathrm{H}, \mathrm{d}, J=6 \mathrm{~Hz}, \mathrm{CH}_{3}-9\right), 1.26-2.34(12 \mathrm{H}, \mathrm{m}), 3.08(1 \mathrm{H}, \mathrm{dd}, J=9,17 \mathrm{~Hz}$, isoxazole-H-4), 3.52 (1H, dd, $J=9,17 \mathrm{~Hz}$, isoxazole-H-4'), 3.65 (1H, dd, $J=9,17 \mathrm{~Hz}$, isoxazole-H4'), 4.00 (1H, dd, $J=10,17 \mathrm{~Hz}$, isoxazole-H-4), 5.51 (1H, m, isoxazole-H-5'), 5.60 (1H, dd, $J=8,10$ $\mathrm{Hz}$, isoxazole-H-5), 5.64 (2H, s, H-12), 7.45 (5H, m, Ph); ${ }^{13} \mathrm{C}-\mathrm{NMR}\left(100 \mathrm{MHz}, \mathrm{CDCl}_{3}, \delta\right): 19.8\left(\mathrm{CH}_{3}\right)$, $23.9\left(\mathrm{CH}_{3}\right), 24.6\left(\mathrm{CH}_{2}\right), 25.5\left(\mathrm{CH}_{2}\right), 25.7\left(\mathrm{CH}_{3}\right), 32.4\left(\mathrm{CH}_{2}\right), 37.0(\mathrm{CH}), 37.1\left(\mathrm{CH}_{2}\right), 43.3\left(\mathrm{CH}_{2}\right), 48.4$ (CH), $52.1(\mathrm{CH}), 82.9(\mathrm{CH}), 83.2(\mathrm{C}), 96.4(\mathrm{CH}), 103.3(\mathrm{C}), 126.9(2 \mathrm{xCH}), 127.9(\mathrm{CH}), 128.4(2 \times \mathrm{CH})$, 166.5 (C). Minor isomer: ${ }^{1} \mathrm{H}-\mathrm{NMR}\left(400 \mathrm{MHz}, \mathrm{CDCl}_{3}, \delta\right): 0.99\left(6 \mathrm{H}, \mathrm{d}, J=6 \mathrm{~Hz}, \mathrm{CH}_{3}-9\right), 1.26-2.34$ 
(12H, m), 3.08 (1H, dd, $J=9,17 \mathrm{~Hz}$, isoxazole-H-4), 3.52 (1H, dd, $J=9,17 \mathrm{~Hz}$, isoxazole-H-4'), 3.65 (1H, dd, $J=9,17 \mathrm{~Hz}$, isoxazole-H-4'), 4.00 (1H, dd, $J=10,17 \mathrm{~Hz}$, isoxazole-H-4), 5.51 (1H, m, isoxazole-H-5'), $5.60\left(1 \mathrm{H}, \mathrm{dd}, J=8,10 \mathrm{~Hz}\right.$, isoxazole-H-5), $5.66(2 \mathrm{H}, \mathrm{s}, \mathrm{H}-12), 7.34(5 \mathrm{H}, \mathrm{m}, \mathrm{Ph}) ;{ }^{13} \mathrm{C}-$ NMR (100 MHz, $\left.\mathrm{CDCl}_{3}, \delta\right): 19.8\left(\mathrm{CH}_{3}\right), 23.5\left(\mathrm{CH}_{3}\right), 24.6\left(\mathrm{CH}_{2}\right), 25.5\left(\mathrm{CH}_{2}\right), 25.7\left(\mathrm{CH}_{3}\right), 32.4\left(\mathrm{CH}_{2}\right)$, $37.0(\mathrm{CH}), 37.1\left(\mathrm{CH}_{2}\right), 43.3\left(\mathrm{CH}_{2}\right), 48.3(\mathrm{CH}), 52.5(\mathrm{CH}), 82.1(\mathrm{CH}), 83.2(\mathrm{C}), 96.4(\mathrm{CH}), 103.3(\mathrm{C})$, $125.9(2 x C H), 127.9(\mathrm{CH}), 128.6(2 x \mathrm{CH}), 166.5(\mathrm{C})$.

9- $\alpha$-(5-Phenyl-3-isoxazol-3-yl)-(1S,4R,5S,8R,9S,11S,12S)-1,5,9-trimethyl-10,13,14,15-tetraoxatetracyclo[9.1.3.1.0 $\left.0^{4,12} \cdot 0^{8,12}\right]$ pentadecane (12)

To the oxime 10 (200 mg, $0.67 \mathrm{mmol})$ in $\mathrm{CH}_{2} \mathrm{Cl}_{2}(20 \mathrm{~mL})$ was added phenylacetylene $(0.75 \mathrm{~mL}$, $6.7 \mathrm{mmol}$ ), a solution of sodium hypochlorite ( $2 \mathrm{~mL}, 13 \%$ active chlorine, $28 \mathrm{mmol}$ ) and a few drops of triethylamine. The mixture was stirred overnight at room temperature and afterwards washed with water (2 times $10 \mathrm{~mL}$ ). The organic layer was dried $\left(\mathrm{MgSO}_{4}\right)$ and evaporated to give a residue that was chromatographed over silica gel with 9/1 dichloromethane/ethyl acetate as the eluent. The product was obtained as a yellow oil (0.055 g, $21 \%$ ); MS (ES): $398\left(\mathrm{M}^{+}\right) ;{ }^{1} \mathrm{H}-\mathrm{NMR}\left(300 \mathrm{MHz}, \mathrm{CDCl}_{3}, \delta\right): 0.99$ (3H, d, $\left.J=6 \mathrm{~Hz}, \mathrm{CH}_{3}-9\right), 1.26-2.34(18 \mathrm{H}, \mathrm{m}), 5.77$ (1H, s, H-12), 6.86 (1H, s, isoxazole-H), $7.41-$ $7.70(5 \mathrm{H}, \mathrm{m}, \mathrm{Ph}) ;{ }^{13} \mathrm{C}-\mathrm{NMR}\left(75 \mathrm{MHz}, \mathrm{CDCl}_{3}, \delta\right): 14.5\left(\mathrm{CH}_{3}\right), 19.8\left(\mathrm{CH}_{3}\right), 25.4\left(\mathrm{CH}_{2}\right), 25.6\left(\mathrm{CH}_{2}\right)$, $25.7\left(\mathrm{CH}_{3}\right), 32.3\left(\mathrm{CH}_{2}\right), 36.9(\mathrm{CH}), 37.0\left(\mathrm{CH}_{2}\right), 48.3(\mathrm{CH}), 52.7(\mathrm{CH}), 82.5(\mathrm{C}), 86.7(\mathrm{C}), 96.5(\mathrm{CH})$, 98.6 (CH), $103.1(\mathrm{C}), 125.7$ (2xCH), $128.0(\mathrm{CH}), 128.7$ (2xCH), $129.6(\mathrm{C}), 168.1(\mathrm{C}), 172.5(\mathrm{C})$.

2'-[(1S,4R,5S,8R,9S,11S,12S)-1,5,9-trimethyl-10,13,14,15-tetraoxatetracyclo[9.1.3.1.0 $\left.0^{4,12} \cdot 0^{8,12}\right]$ penta-

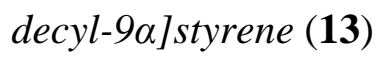

To benzylidene triphenylphosphonium bromide (3.10 g, $7.10 \mathrm{mmol}$ ) in THF $(20 \mathrm{~mL})$ at $-78{ }^{\circ} \mathrm{C}$ was added butyllithium (2M in hexane, $7.10 \mathrm{mmol}$ ). The mixture was stirred for one hour, brought to room temperature and the aldehyde $7(0.5 \mathrm{~g}, 1.77 \mathrm{mmol})$ in THF $(5 \mathrm{~mL})$ was added dropwise. The mixture was stirred one hour under reflux conditions. Afterwards, the mixture was diluted with pentane (20 $\mathrm{mL}$ ) and the resulting precipitate was removed by filtration. The organic layer was evaporated to give a residue that was chromatographed over silica gel with 85:15 $\mathrm{CH}_{2} \mathrm{Cl}_{2}$ /petroleum ether as the eluent. The product was obtained as a yellow oil (0.40 g, $64 \%$ ); MS (ES): $357\left(\mathrm{M}^{+}\right)$; ${ }^{1} \mathrm{H}-\mathrm{NMR}$ (300 MHz, $\left.\mathrm{CDCl}_{3}, \delta\right): 0.99$ (3H, d, $\left.J=6 \mathrm{~Hz}, \mathrm{CH}_{3}-9\right), 1.26-2.34(18 \mathrm{H}, \mathrm{m}), 5.72(1 \mathrm{H}, \mathrm{s}, \mathrm{H}-12), 6.51$ (1H, d, $J=16$ $\mathrm{Hz}, \mathrm{CH}=\mathrm{CH}-\mathrm{Ph}), 6.78(1 \mathrm{H}, \mathrm{d}, J=16 \mathrm{~Hz}, \mathrm{C} \underline{\mathbf{H}}=\mathrm{CH}-\mathrm{Ph}), 7.27(5 \mathrm{H}, \mathrm{m}, \mathrm{Ph}) ;{ }^{13} \mathrm{C}-\mathrm{NMR}\left(75 \mathrm{MHz}, \mathrm{CDCl}_{3}\right.$,

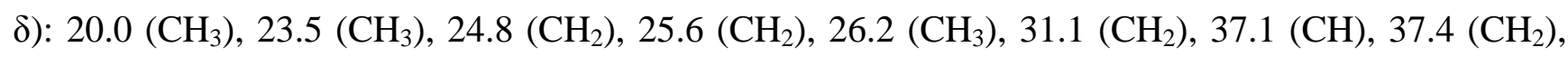
$48.0(\mathrm{CH}), 53.6(\mathrm{CH}), 85.8(\mathrm{C}), 87.0(\mathrm{C}), 96.8(\mathrm{CH}), 103.5(\mathrm{C}), 125.9(\mathrm{CH}), 126.7(\mathrm{CH}), 127.0$ (2xCH), $128.5(2 \times C H), 137.9(\mathrm{CH}), 138.3(\mathrm{C})$

Ethyl-2-[(1S,4R,5S,8R,9S,11S,12S)-1,5,9-trimethyl-10,13,14,15-tetraoxatetracyclo $\left[9.1 \cdot 3 \cdot 1 \cdot 0^{4,12} \cdot 0^{8,12}\right]$ -

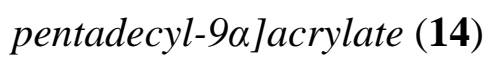

To the aldehyde 7 (0.5 g, $1.77 \mathrm{mmol})$ in THF (15 mL) was added carbethoxymethylene triphenylphosphorane (2.35 g, $6.80 \mathrm{mmol})$. The mixture was stirred overnight at room temperature. 
The organic layer was evaporated to give a residue that was chromatographed over silica gel with 9:1 $\mathrm{CH}_{2} \mathrm{Cl}_{2}$ /ethylacetate as the eluent. The product was obtained as white crystals (0.46 g, $77 \%$ ); MS (ES): $353\left(\mathrm{M}^{+}\right)$; mp: $135-136{ }^{\circ} \mathrm{C}$; ${ }^{1} \mathrm{H}-\mathrm{NMR}\left(300 \mathrm{MHz}, \mathrm{CDCl}_{3}, \delta\right.$ ): 0.99 (3H, d, $\left.J=6 \mathrm{~Hz}, \mathrm{CH}_{3} 9\right)$, 1.26$2.34(21 \mathrm{H}, \mathrm{m}), 4.16\left(2 \mathrm{H}, \mathrm{m}, \mathrm{COOC} \underline{\mathbf{H}}_{2} \mathrm{CH}_{3}\right), 5.69(1 \mathrm{H}, \mathrm{s}, \mathrm{H}-12), 6.06(1 \mathrm{H}, \mathrm{d}, J=16 \mathrm{~Hz}, \mathrm{CH}=\mathrm{C} \underline{\mathbf{H}} \mathrm{CO})$, $7.22(1 \mathrm{H}, \mathrm{d}, \quad J=16 \mathrm{~Hz}, \mathrm{C} \underline{\mathbf{H}}=\mathrm{CHCO}) ;{ }^{13} \mathrm{C}-\mathrm{NMR}\left(75 \mathrm{MHz}, \mathrm{CDCl}_{3}, \delta\right): 14.4\left(\mathrm{CH}_{3}\right), 19.9\left(\mathrm{CH}_{3}\right), 23.4$ $\left(\mathrm{CH}_{3}\right), 24.8\left(\mathrm{CH}_{2}\right), 25.5\left(\mathrm{CH}_{2}\right), 25.8\left(\mathrm{CH}_{3}\right), 32.7\left(\mathrm{CH}_{2}\right), 37.0(\mathrm{CH}), 37.2\left(\mathrm{CH}_{2}\right), 48.7(\mathrm{CH}), 52.7(\mathrm{CH})$, $60.3\left(\mathrm{CH}_{2}\right), 85.1(\mathrm{C}), 86.4(\mathrm{C}), 96.8(\mathrm{CH}), 103.5(\mathrm{C}), 117.7(\mathrm{CH}), 154.3(\mathrm{CH}), 167.3(\mathrm{CO})$

3-[(1S,4R,5S,8R,9S,11S,12S)-1,5,9-trimethyl-10,13,14,15-tetraoxatetracyclo[9.1.3.1.0 $\left.0^{4,12} \cdot 0^{8,12}\right]$ -

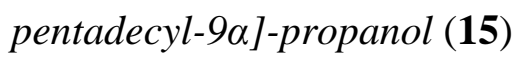

To the aldehyde 7 (0.025 g, $0.0087 \mathrm{mmol})$ in THF (5 mL) was added 5\% Pd/C-catalyst $(0.010 \mathrm{~g})$. Air was removed and hydrogen was added. The mixture was stirred overnight, then filtered over Celite ${ }^{\circledR}$. The organic layer was dilute with diethyl ether $(5 \mathrm{~mL})$, washed with brine, dried $\left(\mathrm{MgSO}_{4}\right)$ and evaporated to give a residue that was chromatographed over silica gel with 9:1 $\mathrm{CH}_{2} \mathrm{Cl}_{2}$ /ethyl acetate as the eluent. The product was obtained as yellow oil (0.020 g, $74 \%$ ); MS (ES): $313\left(\mathrm{M}^{+}\right) ;{ }^{1} \mathrm{H}-$ NMR (300 MHz, $\mathrm{CDCl}_{3}, \delta$ ): 0.90 (2H, m, $\mathrm{CH}_{2}-10$ ), 0.91 (3H, d, $J=6 \mathrm{~Hz}, \mathrm{CH}_{3}-9$ ), 1.26-2.34 (20H, m), 4.21 (2H, dd, J=2, $\left.6 \mathrm{~Hz}, \underline{\mathrm{C}}_{2}-\mathrm{OH}\right), 5.62(1 \mathrm{H}, \mathrm{s}, \mathrm{H}-12) ;{ }^{13} \mathrm{C}-\mathrm{NMR}\left(75 \mathrm{MHz}, \mathrm{CDCl}_{3}, \delta\right): 18.7\left(\mathrm{CH}_{3}\right)$, $22.0\left(\mathrm{CH}_{3}\right), 24.0\left(\mathrm{CH}_{2}\right), 25.6\left(\mathrm{CH}_{2}\right), 26.5\left(\mathrm{CH}_{3}\right), 29.4\left(\mathrm{CH}_{2}\right), 31.0\left(\mathrm{CH}_{2}\right), 33.0\left(\mathrm{CH}_{2}\right), 35.2(\mathrm{CH}), 36.2$ $\left(\mathrm{CH}_{2}\right), 46.8(\mathrm{CH}), 51.1(\mathrm{CH}), 60.6\left(\mathrm{CH}_{2}\right), 86.5(\mathrm{C}), 89.3(\mathrm{C}), 94.2(\mathrm{CH}), 103.7(\mathrm{C})$

(1S,4R,5S,8R,9S,11S,12S)-1,5,9-trimethyl-10,13,14,15-tetraoxatetracyclo[9.1.3.1.0 $\left.0^{4,12} \cdot 0^{8,12}\right]$ penta-

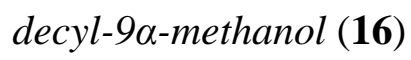

To the aldehyde $7(0.050 \mathrm{~g}, 0.18 \mathrm{mmol})$ in methanol $(5 \mathrm{~mL})$ was added sodium borohydride $(0.027$ g, $0.72 \mathrm{mmol})$ in small portions. The mixture was stirred for one hour at $0{ }^{\circ} \mathrm{C}$. Afterwards, $\mathrm{HCl}(0.2$ $\mathrm{mL}, 7.5 \mathrm{~N}$ ) was added in a dropwise manner and the mixture was stirred for 30 minutes and then evaporated. The residue was diluted with water $(10 \mathrm{~mL})$ and extracted with diethyl ether $(3 \mathrm{x} 15 \mathrm{~mL})$. The organic layer was washed with $\mathrm{NaHCO}_{3}$, dried $\left(\mathrm{MgSO}_{4}\right)$ and evaporated. The product was obtained as white crystals (0.042 g, $84 \%$ ); MS (ES): $285\left(\mathrm{M}^{+}\right)$; mp: $123{ }^{\circ} \mathrm{C}$; ${ }^{1} \mathrm{H}-\mathrm{NMR}(300 \mathrm{MHz}$, $\left.\mathrm{CDCl}_{3}, \delta\right): 0.99\left(3 \mathrm{H}, \mathrm{d}, J=6 \mathrm{~Hz}, \mathrm{CH}_{3}-9\right), 1.26-2.34(18 \mathrm{H}, \mathrm{m}), 3.55\left(1 \mathrm{H}, J=8,11 \mathrm{~Hz}, \mathrm{C}_{2} \mathrm{OH}\right), 4.04$ (1H, dd, $\left.J=8,11 \mathrm{~Hz}, \underline{\mathrm{C}}_{2} \mathrm{OH}\right), 5.61(1 \mathrm{H}, \mathrm{s}, \mathrm{H}-12) ;{ }^{13} \mathrm{C}-\mathrm{NMR}$ (75 MHz, $\left.\mathrm{CDCl}_{3}, \delta\right): 20.1\left(\mathrm{CH}_{3}\right), 20.3$ $\left(\mathrm{CH}_{3}\right), 24.4\left(\mathrm{CH}_{2}\right), 25.5\left(\mathrm{CH}_{2}\right), 26.2\left(\mathrm{CH}_{3}\right), 32.8\left(\mathrm{CH}_{2}\right), 36.9(\mathrm{CH}), 37.4\left(\mathrm{CH}_{2}\right), 48.1(\mathrm{CH}), 49.3(\mathrm{CH})$, $69.2\left(\mathrm{CH}_{2}\right), 85.4(\mathrm{C}), 89.8(\mathrm{C}), 96.2(\mathrm{CH}), 103.7(\mathrm{C})$

O-[(1S,4R,5S,8R,9S,11S,12S)-1,5,9-trimethyl-10,13,14,15-tetraoxatetracyclo[9.1.3.1.0 $\left.0^{4,12} \cdot 0^{8,12}\right]$ pentadecyl-9 $\alpha$-methyl]-N-phenylcarbamate (17)

To the alcohol $16(0.082 \mathrm{~g}, 0.29 \mathrm{mmol})$ in pyridine $(5 \mathrm{~mL})$ was added phenyl isocyanate $(0.078$ $\mathrm{mL}, 0.72 \mathrm{mmol})$. The mixture was stirred for 14 hours by $60{ }^{\circ} \mathrm{C}$. Afterwards, the mixture was extracted with petroleum ether $(3 \times 10 \mathrm{~mL})$. The organic layer was washed with $\mathrm{HCl}(1 \mathrm{~N})$ and brine, dried $\left(\mathrm{MgSO}_{4}\right)$ and evaporated to give a residue that was chromatographed over silica gel with 95:5 
$\mathrm{CH}_{2} \mathrm{Cl}_{2}$ /ethyl acetate as the eluent. The product was obtained as an oil (0.070 g, 67 \%); MS (ES): 404 $\left(\mathrm{M}^{+}\right)$; ${ }^{1} \mathrm{H}-\mathrm{NMR}\left(300 \mathrm{MHz}, \mathrm{CDCl}_{3}, \delta\right.$ ): 0.99 (3H, d, $\left.J=6, \mathrm{CH}_{3}-9\right), 1.26-2.34$ (18H, m), 4.50 (2H, dd, $J=$ 10, $\left.11 \mathrm{~Hz}, \underline{\mathrm{C}}_{2} \mathrm{COONHPhH}\right), 5.67(1 \mathrm{H}, \mathrm{s}, \mathrm{H}-12), 6.80-7.40$ (5H, m, Ph); ${ }^{13} \mathrm{C}-\mathrm{NMR}\left(75 \mathrm{MHz}, \mathrm{CDCl}_{3}\right.$,

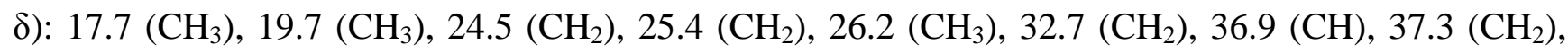
$48.7(\mathrm{CH}), 49.0(\mathrm{CH}), 73.6\left(\mathrm{CH}_{2}\right), 84.3(\mathrm{C}), 86.6(\mathrm{C}), 96.6(\mathrm{CH}), 103.8(\mathrm{C}), 121.3(\mathrm{CH}), 122.3$ (2XCH), $123.4(2 \times C H), 129.2(\mathrm{C}), 138.1(\mathrm{CO})$

\section{$O-\left[(1 S, 4 R, 5 S, 8 R, 9 S, 11 S, 12 S)-1,5,9-\right.$ trimethyl-10,13,14,15-tetraoxatetracyclo[9.1.3.1.0 $\left.0^{4,12} \cdot 0^{8,12}\right]$ -} pentadecyl-9 $\alpha$-methyl]-N-allylthiocarbamate (18)

To the alcohol $16(0.58 \mathrm{~g}, 2.04 \mathrm{mmol})$ in pyridine $(25 \mathrm{~mL})$ was added allyl isothiocyanate $(0.50 \mathrm{~g}$, $5.09 \mathrm{mmol}$ ). The mixture was stirred for 14 hours by $60^{\circ} \mathrm{C}$ and then extracted with petroleum ether ( 3 x $30 \mathrm{~mL})$. The organic layer was washed with $\mathrm{HCl}(1 \mathrm{~N})$ and brine, dried $\left(\mathrm{MgSO}_{4}\right)$ and evaporated to give a residue that was chromatographed over silica gel with $95: 5 \mathrm{CH}_{2} \mathrm{Cl}_{2}$ /ethyl acetate as the eluent. The product was obtained as an oil (0.42 g, 53 \%); MS (ES): $384\left(\mathrm{M}^{+}\right)$; ${ }^{1} \mathrm{H}-\mathrm{NMR}$ (300 MHz, $\mathrm{CDCl}_{3}$,

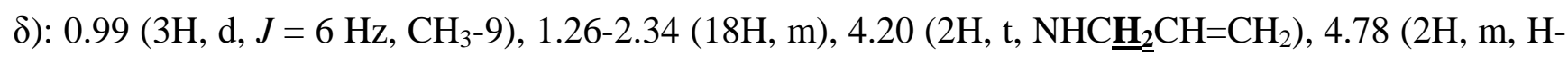
10), $5.22\left(2 \mathrm{H}, \mathrm{m}, \mathrm{NHCH}_{2} \mathrm{CH}=\underline{\mathrm{C}}_{2}\right), 5.65(1 \mathrm{H}, \mathrm{s}, \mathrm{H}-12), 5.87\left(1 \mathrm{H}, \mathrm{m}, \mathrm{NHCH}_{2} \mathrm{C} \underline{\mathbf{H}}=\mathrm{CH}_{2}\right), 6.45(1 \mathrm{H}, \mathrm{s}$, $\mathrm{NH}) ;{ }^{13} \mathrm{C}-\mathrm{NMR}\left(75 \mathrm{MHz}, \mathrm{CDCl}_{3}, \delta\right): 17.7\left(\mathrm{CH}_{3}\right), 19.7\left(\mathrm{CH}_{3}\right), 24.6\left(\mathrm{CH}_{2}\right), 24.8\left(\mathrm{CH}_{2}\right), 25.4\left(\mathrm{CH}_{3}\right), 32.4$ $\left(\mathrm{CH}_{2}\right), 37.0(\mathrm{CH}), 37.2\left(\mathrm{CH}_{2}\right), 47.9(\mathrm{CH}), 49.0(\mathrm{CH}), 49.1\left(\mathrm{CH}_{2}\right), 75.5\left(\mathrm{CH}_{2}\right), 85.5(\mathrm{C}), 86.5(\mathrm{C}), 96.6$ (CH), $103.8(\mathrm{C}), 117.5\left(\mathrm{CH}_{2}\right), 132.8(\mathrm{CH}), 157.9(\mathrm{CS})$

\section{$N$-(4-methylphenyl)-(1S,4R,5S,8R,9S,11S,12S)-1,5,9-trimethyl-10,13,14,15-tetraoxatetracyclo} [9.1.3.1. $\left.0^{4,12} \cdot 0^{8,12}\right]$-pentadecyl-9 $\alpha$-methylamine (19)

To the aldehyde 7 (0.5 g, $1.77 \mathrm{mmol})$ in methanol $(15 \mathrm{~mL})$ was added p-toluidine (0.79 g, 7.08 $\mathrm{mmol})$ and sodium cyanoborohydride $(0.44 \mathrm{~g}, 7.08 \mathrm{mmol})$. The mixture was stirred for 30 minutes at room temperature, afterwards $\mathrm{KOH}(0.4 \mathrm{~g}, 7.08 \mathrm{mmol})$ was added in small portions and stirring continued for 30 minutes. The mixture was diluted with $\mathrm{NH}_{4} \mathrm{Cl}$-solution $(10 \mathrm{~mL})$ and extracted with ethyl acetate $(3 \times 15 \mathrm{~mL})$. The organic layer was washed with brine, dried $\left(\mathrm{MgSO}_{4}\right)$ and evaporated. The residue was chromatographed over silica gel with 95:5 $\mathrm{CH}_{2} \mathrm{Cl}_{2}$ /ethyl acetate as the eluent. The product was obtained as a yellow oil (0.49 g, $74 \%$ ); MS (ES): $374\left(\mathrm{M}^{+}\right) ;{ }^{1} \mathrm{H}-\mathrm{NMR}$ (300 MHz, $\mathrm{CDCl}_{3}$, $\delta$ ): 0.98 (3H, d, $\left.J=6 \mathrm{~Hz}, \mathrm{CH}_{3}-9\right), 1.26-2.34(21 \mathrm{H}, \mathrm{m}), 3.43\left(1 \mathrm{H}, \mathrm{dd}, J=4,12 \mathrm{~Hz}, \mathrm{C}_{2}-10\right), 3.58(1 \mathrm{H}$, dd, $\left.J=3,13 \mathrm{~Hz}, \underline{\mathrm{C}}_{2}-10\right), 5.63$ (1H, s, H-12), 6.66 (2H, d, $\left.J=8 \mathrm{~Hz}, \mathrm{o}-\mathrm{Ph}\right), 6.97$ (2H, d, $J=8 \mathrm{~Hz}, \mathrm{~m}-$ $\mathrm{Ph}) ;{ }^{13} \mathrm{C}-\mathrm{NMR}$ (75 MHz, $\left.\mathrm{CDCl}_{3}, \delta\right): 14.3\left(\mathrm{CH}_{3}\right), 21.2\left(\mathrm{CH}_{3}\right), 24.4\left(\mathrm{CH}_{2}\right), 25.5\left(\mathrm{CH}_{2}\right), 26.4\left(\mathrm{CH}_{3}\right), 31.0$ $\left(\mathrm{CH}_{2}\right), 36.9(\mathrm{CH}), 37.4\left(\mathrm{CH}_{2}\right), 47.3(\mathrm{CH}), 53.6(\mathrm{CH}), 60.5\left(\mathrm{CH}_{2}\right), 85.2(\mathrm{C}), 87.0(\mathrm{C}), 96.3(\mathrm{CH}), 103.8$ (C), 113.7 (2xCH), $117.6\left(\mathrm{CH}_{3}\right), 120.7(\mathrm{C}), 129.8(2 \times C H), 171.3(\mathrm{C})$

$(1 S, 4 R, 5 S, 8 R, 9 S, 11 S, 12 S)-1,5,9$-trimethyl-10,13,14,15-tetraoxatetracyclo[9.1.3.1.0 $\left.0^{4,12} \cdot 0^{8,12}\right]$ pentadecyl-9 $\alpha$-acid (20)

To the aldehyde $7(0.250 \mathrm{~g}, 0.87 \mathrm{mmol})$ in 1,4-dioxane $(10 \mathrm{~mL})$ was added aminosulfonic acid ( $0.34 \mathrm{~g}, 3.55 \mathrm{mmol})$ and water $(3.5 \mathrm{~mL})$. The mixture was stirred for 20 hours at $0{ }^{\circ} \mathrm{C}$, then $\mathrm{NaClO}_{2}$ 
(0.32 g, $3.55 \mathrm{mmol})$ and water $(2.5 \mathrm{~mL})$ were added. After stirring for 30 minutes $\mathrm{Na}_{2} \mathrm{SO}_{4}(0.250 \mathrm{~g})$ was added and the mixture was diluted with water $(10 \mathrm{~mL})$. The mixture was extracted with diethyl ether (3 x $20 \mathrm{~mL})$ and the organic layer was washed with brine, dried $\left(\mathrm{MgSO}_{4}\right)$ and evaporated. The product was obtained as a brown solid (0.225 mg, $80 \%$ ); mp: $137{ }^{\circ} \mathrm{C} ;{ }^{1} \mathrm{H}-\mathrm{NMR}\left(300 \mathrm{MHz}, \mathrm{CDCl}_{3}, \delta\right.$ ): 1.01 (3H, d, $\left.J=6 \mathrm{~Hz}, \mathrm{CH}_{3}-9\right)$, 1.26-2.34 (18H, m), 5.72 (1H, s, H-12), 9.47 (1H, s, COO杰); ${ }^{13} \mathrm{C}-\mathrm{NMR}$ (75 MHz, $\left.\mathrm{CDCl}_{3}, \delta\right): 19.9\left(\mathrm{CH}_{3}\right), 21.1\left(\mathrm{CH}_{3}\right), 24.6\left(\mathrm{CH}_{2}\right), 25.0\left(\mathrm{CH}_{2}\right), 25.7\left(\mathrm{CH}_{3}\right), 32.4\left(\mathrm{CH}_{2}\right), 36.7$ $(\mathrm{CH}), 37.2\left(\mathrm{CH}_{2}\right), 47.6(\mathrm{CH}), 50.5(\mathrm{CH}), 85.4(\mathrm{C}), 86.7(\mathrm{C}), 97.6(\mathrm{CH}), 103.7(\mathrm{C}), 175.6(\mathrm{CO})$

1 '-[(1S,4R,5S,8R,9S,11S,12S)-1,5,9-trimethyl-10,13,14,15-tetraoxatetracyclo[9.1.3.1.0 $\left.0^{4,12} \cdot 0^{8,12}\right]$ penta-

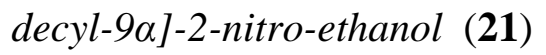

To the aldehyde $7(0.050 \mathrm{~g}, 0.18 \mathrm{mmol})$ in ethanol $(5 \mathrm{~mL})$ was added nitromethane $(0.038 \mathrm{~mL}$, $0.72 \mathrm{mmol})$, the mixture was cooled to $0{ }^{\circ} \mathrm{C}$ and sodium methoxide $(1 \mathrm{~g})$ in methanol $(5 \mathrm{~mL})$ was added dropwise. The mixture was stirred for 18 minutes at $0{ }^{\circ} \mathrm{C}$, then diluted with ethyl acetate and washed with brine. The organic layer was dried and evaporated and the product was obtained as an oil (0.040 g, 67 \%); ${ }^{1} \mathrm{H}-\mathrm{NMR}$ (300 MHz, $\mathrm{CDCl}_{3}, \delta$ ): 0.99 (3H, d, $J=6 \mathrm{~Hz}, \mathrm{CH}_{3}-9$ ), 1.15 (3H, s, $\mathrm{CH}_{3}-6$ ), 1.26-2.34 (15H, m), 4.44 (1H, dd, $J=4,10 \mathrm{~Hz}, \mathrm{H}-10), 4.93\left(1 \mathrm{H}, \mathrm{dd}, J=4,10 \mathrm{~Hz}, \mathrm{C}_{2} \mathrm{NO}_{2}\right), 5.12(1 \mathrm{H}$, dd, $\left.J=4,10 \mathrm{~Hz}, \underline{\mathrm{H}}_{2} \mathrm{NO}_{2}\right), 5.59(1 \mathrm{H}, \mathrm{s}, \mathrm{H}-12) ;{ }^{13} \mathrm{C}-\mathrm{NMR}\left(75 \mathrm{MHz}, \mathrm{CDCl}_{3}, \delta\right): 16.6\left(\mathrm{CH}_{3}\right), 20.0$ $\left(\mathrm{CH}_{3}\right), 24.4\left(\mathrm{CH}_{2}\right), 25.3\left(\mathrm{CH}_{2}\right), 26.3\left(\mathrm{CH}_{3}\right), 32.7\left(\mathrm{CH}_{2}\right), 36.9(\mathrm{CH}), 47.6(\mathrm{CH}), 49.1(\mathrm{CH}), 60.6(\mathrm{CH})$, $72.4\left(\mathrm{CH}_{2}\right), 85.1(\mathrm{C}), 86.9(\mathrm{C}), 96.6(\mathrm{CH}), 103.9(\mathrm{C})$

(1S,4R,5S,8R,9S,11S,12S)-1,5,9-trimethyl-10,13,14,15-tetraoxatetracyclo[9.1.3.1.0 $\left.0^{4,12} \cdot 0^{8,12}\right]$ pentadecyl-9 $\alpha$-2'-aminoacetonitrile (22)

To the aldehyde 7 ( $1.4 \mathrm{~g}, 4.90 \mathrm{mmol})$ in methanol $(25 \mathrm{~mL})$ was added a solution of sodium cyanide (0.98 g, $20 \mathrm{mmol})$ in water $(25 \mathrm{~mL})$ and $\mathrm{NH}_{4} \mathrm{Cl}$. The mixture was stirred for 2 hours at $45{ }^{\circ} \mathrm{C}$. Afterwards the mixture was diluted with water $(10 \mathrm{~mL})$ and extracted with toluene $(3 \times 50 \mathrm{~mL})$. The organic layer was washed with brine, dried $\left(\mathrm{MgSO}_{4}\right)$ and evaporated. The product was obtained as an oil (1.2 g, 78 \%); MS (ES): $309\left(\mathrm{M}^{+}\right)$; ${ }^{1} \mathrm{H}-\mathrm{NMR}$ (300 MHz, $\mathrm{CDCl}_{3}, \delta$ ): 1.01 (3H, d, $J=6 \mathrm{~Hz}, \mathrm{CH}_{3}-9$ ), 1.26-2.34 (18H, m), 5.17 (1H, s, H-10), 5.63 (1H, s, H-12); ${ }^{13} \mathrm{C}-\mathrm{NMR}\left(75 \mathrm{MHz}, \mathrm{CDCl}_{3}, \delta\right): 18.2$ $\left(\mathrm{CH}_{3}\right), 19.8\left(\mathrm{CH}_{3}\right), 24.2\left(\mathrm{CH}_{2}\right), 25.2\left(\mathrm{CH}_{2}\right), 25.8\left(\mathrm{CH}_{3}\right), 32.5\left(\mathrm{CH}_{2}\right), 37.1\left(\mathrm{CH}_{2}\right), 47.2(\mathrm{CH}), 48.6(\mathrm{CH})$, $48.9(\mathrm{CH}), 84.6(\mathrm{C}), 86.9(\mathrm{C}), 96.7(\mathrm{CH}), 103.9(\mathrm{C})$

(1S,4R,5S,8R,9S,11S,12S)-1,5,9-trimethyl-10,13,14,15-tetraoxatetracyclo[9.1.3.1.0 $\left.0^{4,12} \cdot 0^{8,12}\right]$ pentadecyl-9 $\alpha$-2'-hydroxyacetonitrile (23)

To the aldehyde $7(0.050 \mathrm{~g}, 0.18 \mathrm{mmol})$ in methanol $(5 \mathrm{~mL})$ was added acetic acid $(0.10 \mathrm{~mL}, 70 \%)$ and potassium cyanide $(0.046 \mathrm{~g}, 0.71 \mathrm{mmol})$. The mixture was stirred for 18 hours at room temperature and then evaporated to give a residue that was diluted with ethyl acetate $(10 \mathrm{~mL})$ and the resulting precipitate was removed by filtration. The organic layer was evaporated to give a residue that was chromatographed over silica gel with 9:1 ethyl acetate/methanol as the eluent. The product was obtained as an oil (0.045 g, 83 \%); MS (ES): $310\left(\mathrm{M}^{+}\right) ;{ }^{1} \mathrm{H}-\mathrm{NMR}$ (300 MHz, $\mathrm{CDCl}_{3}, \delta$ ): 1.00 (3H, d, $J$ 
$\left.=6 \mathrm{~Hz}, \mathrm{CH}_{3}-9\right), 1.26-2.34(18 \mathrm{H}, \mathrm{m}), 5.16(1 \mathrm{H}, \mathrm{s}, \mathrm{H}-10), 5.62(1 \mathrm{H}, \mathrm{s}, \mathrm{H}-12) ;{ }^{13} \mathrm{C}-\mathrm{NMR}(75 \mathrm{MHz}$, $\left.\mathrm{CDCl}_{3}, \delta\right): 17.8\left(\mathrm{CH}_{3}\right), 19.6\left(\mathrm{CH}_{3}\right), 23.5\left(\mathrm{CH}_{2}\right), 24.9\left(\mathrm{CH}_{2}\right), 25.0\left(\mathrm{CH}_{3}\right), 32.2\left(\mathrm{CH}_{2}\right), 36.30(\mathrm{CH}), 36.7$ $\left(\mathrm{CH}_{2}\right), 46.6(\mathrm{CH}), 48.5(\mathrm{CH}), 86.6(\mathrm{C}), 89.2(\mathrm{C}), 95.9(\mathrm{CH}), 103.0(\mathrm{C})$

\section{Acknowledgements}

We wish to acknowledge financial support from the University of Leuven and the F. W. O.Vlaanderen. We thank Dr F. H. Jansen (DAFRA) for a generous gift of artemisinin.

\section{References and Notes}

1. For recent review articles on artemisinin and its derivatives see: (a) Kim B.J.; Sasaki, T. Recent progress in the synthesis of artemisinin and its derivatives Org. Prep. Proced. Int. 2006, 38, 1-80; (b) Jung, M.; Lee, K., Kim, H, Park, M. Recent advances in artemisinin and its derivatives as antimalarial and antitumor agents. Current Med. Chem. 2004, 11, 1265-1284; (c) van Agtmael, M. A.; Eggelte, T. A.; van Boxtel C. J. Artemisinin drugs in the treatment of malaria : from medicinal herb to registered medication Trends Pharm. Sci. 1999, 20, 199-205.

2. Hindley, S.; Ward, S. A.; Storr, R. C.; Searle, N. L.; Bray, P. G.; Park, B. K.; Davies, J.; O’Neill, P. M. Mechanism-based design of parasite-targeted artemisinin derivatives: synthesis and antimalarial activity of new diamine containing analogues. J. Med. Chem. 2002, 45, 1052-1063.

3. (a) Grellepois, F.; Bonnet-Delpon, D.; Bégué, J.-P. Ring-contracted artemisinin derivatives: stereoselective reaction of anhydrodihydroartemisinin towards halogenation reagents. Tetrahedron Lett. 2001, 42, 2125-2127; (b) Grellepois, F.; Chorki, F. Crousse, B.; Ourévitch, M.; Bonnet-Delpon, D.; Bégué, J.-P. Anhydrodihydroartemisin and its 10-trifluoromethyl analogue: access to novel D-ring-contracted artemisinin trifluoromethyl ketones J. Org. Chem. 2002, 67, 1253-1260.

4. (a) Lee, G.A. A simplified synthesis of unsaturated nitrogen heterocycles using nitrilium betaines Synthesis 1982, 508-509; L’abbé, G.; Emmers, S.; Dehaen, W.; Dyall, L. 5-Chloropyrazole-4carbaldehydes as synthons for intramolecular 1,3-dipolar cycloadditions, J. Chem. Soc. Perkin Trans 1 1994, 2553-2558.

5. For a few recent examples see: (a) Liu, Y.; Wong, V. K.-W.; Ko, B. C.-B.;Wong, M.-K.; Che, C.M. Synthesis and cytotoxic study of artemisinin derivatives containing lipophilic alkyl carbon chains Org. Lett. 2005, 7, 1561-1564; (b) Ma, J.; Katz, E.; Kyle, D. E.; Ziffer, H. Syntheses and antimalarial activities of 10-substituted deoxoartemisinins J. Med. Chem. 2000, 43, 4228-4232.

6. Venugopalan, B.; Bapat, C. P.; Karnik, P. J.; Chatterjee, D. K.; Iyer, N.; Lepcha, D. Antimalarial activity of novel ring-contracted artemisinin derivatives. J. Med. Chem. 1995, 38, 1922

Sample Availability: Samples of the compounds described in this article are available from the authors.

(C) 2007 by MDPI (http://www.mdpi.org). Reproduction is permitted for noncommercial purposes. 